\title{
Mobility Limitations due to Dislocations and Interface Roughness in AlGaN/AlN/GaN Heterostructure
}

\author{
Qun Li, ${ }^{1}$ Jingwen Zhang, ${ }^{1}$ Li Meng, ${ }^{1}$ Jing Chong, ${ }^{2}$ and Xun Hou ${ }^{1}$ \\ ${ }^{1}$ Key Laboratory for Physical Electronics and Devices of the Ministry of Education, Key Laboratory of Photonics Technology for \\ Information of Shaanxi Province, and ISCAS-XJTU Joint Laboratory of Functional Materials and Devices for Informatics, \\ School of Electronics and Information Engineering, Xian Jiaotong University, Xian 710049, China \\ ${ }^{2}$ China Satellite Maritime Tracking and Control Department, Jiangyin 214431, China
}

Correspondence should be addressed to Jingwen Zhang; jwzhang@mail.xjtu.edu.cn

Received 12 March 2015; Revised 29 May 2015; Accepted 8 June 2015

Academic Editor: Meiyong Liao

Copyright (C) 2015 Qun Li et al. This is an open access article distributed under the Creative Commons Attribution License, which permits unrestricted use, distribution, and reproduction in any medium, provided the original work is properly cited.

\begin{abstract}
The dislocations and surface roughness in an $\mathrm{AlGaN} / \mathrm{AlN} / \mathrm{GaN}$ heterostructure were analyzed by transmission electron microscopy (TEM) and atomic force microscopy (AFM), respectively, and the mobility limitation mechanisms in the two-dimensional electron gas (2DEG) were studied using a theoretical model that took into account the most important scattering mechanisms. An exponential correlation function provides a better description of the statistical properties of surface roughness than the Gaussian form and thus is adopted in the theoretical model. The calculated results are in good agreement with Hall data. The quantitative measurements of dislocations and surface roughness allow the evaluation of the relative importance of each extrinsic scattering mechanism.
\end{abstract}

\section{Introduction}

GaN-based materials have attracted a great deal of attention over the past two decades due to their direct and large band gap. A high sheet carrier density (over $1.0 \times 10^{13} \mathrm{~cm}^{-2}$ ) and high mobility (over $6.0 \times 10^{4} \mathrm{~cm}^{2} / \mathrm{Vs}$ ) two-dimensional electron gas (2DEG) can be formed in AlGaN/GaN heterostructures even without intentionally doping $[1,2]$, which forecasts the prospects for fabricating high-power, highfrequency microwave devices.

In an ideal, defect free material, the electron mobility is mainly limited by electron-phonon interactions. In a real heterostructure material, however, the extrinsic scattering mechanisms, such as dislocations, interface roughness, and charged impurities, may dominate the electron mobility, particularly at low temperatures.

Most GaN thin films are grown on c-plane sapphire substrates by metal-organic chemical vapor deposition (MOCVD). The larger lattice mismatch between the GaN layer and the sapphire substrate induces a large number of dislocations. Great efforts have been made to improve the crystal quality of $\mathrm{GaN}$ films by preparing a nucleation layer, optimizing growth parameters, and so on, but $\mathrm{GaN}$ still has a dislocation density up to $10^{8} \sim 10^{10} \mathrm{~cm}^{-2}$ [3]. Although highdensity dislocations do not significantly affect the efficiencies of GaN-based light-emitting diodes (LEDs) and laser diodes (LDs) [4], they may dominate the electron mobility in $\mathrm{AlGaN} / \mathrm{GaN}$ heterostructures $[5,6]$.

Interface roughness is another important scattering mechanism that affects the electron mobility in $\mathrm{AlGaN} / \mathrm{GaN}$ heterostructures. Interface roughness is difficult to be measured directly. Cross-sectional transmission electron microscopy (TEM) measurements can give a direct observation of spatial variation in interface structure, but this approach is not convenient to quantify the interface roughness. For the heterostructures grown in a two-dimensional (2D) growth mode, the interface roughness can be approximated by surface roughness and thus can be characterized by atomic force microscopy (AFM) measurements. The effect of interface roughness on electron mobility is also difficult to be precisely modeled due to the random variation of roughness height. In theoretical calculations, a correlation function has usually been adopted to describe the statistical properties of interface roughness for mathematical convenience $[7,8]$. 


\begin{tabular}{|c|}
\hline $20 \mathrm{~nm} \mathrm{Al}_{0.2} \mathrm{Ga}_{0.8} \mathrm{~N}$ barrier layer \\
\hline $1 \mathrm{~nm}$ AlN interfacial layer \\
\hline $100 \mathrm{~nm}$ GaN channel layer \\
\hline $1.5 \mu \mathrm{m}$ semi-insulating GaN layer \\
\hline $120 \mathrm{~nm}$ AlN nucleation layer \\
\hline$c$-sapphire
\end{tabular}

FIGURE 1: Schematic drawing of the Ga-polar AlGaN/AlN/GaN heterostructure grown by MOCVD on $c$-sapphire substrate.

In addition to dislocations and interface roughness, charged residual impurities and alloy disorder may play important roles in the electron transport in a 2DEG system [9, 10]. In most theoretical studies regarding electron transport, the parameters describing extrinsic scattering mechanisms, such as dislocation density and impurity density, have been treated as adjustable fitting parameters [11, 12]; however, the relative importance of different extrinsic scattering mechanisms cannot be evaluated when the number of the fitting parameters is more than one. To evaluate their relative importance with regard to the mobility limitation, it is necessary first to quantify these parameters via direct measurements and then to calculate the mobility limited by individual scattering mechanisms.

In this work we prepared an $\mathrm{AlGaN} / \mathrm{AlN} / \mathrm{GaN}$ heterostructure by MOCVD on $c$-sapphire and studied the mobility limitation mechanisms in the 2DEG. The TEM and AFM techniques are used to analyze the dislocations and surface roughness in the $\mathrm{AlGaN} / \mathrm{AlN} / \mathrm{GaN}$ heterostructure, respectively. The measured results of dislocations and surface roughness are put into a theoretical model that takes into account the most important scattering mechanisms to fit the Hall data and to evaluate the relative importance of each extrinsic scattering mechanism.

\section{Experimental Results}

A Ga-polar AlGaN/AlN/GaN heterostructure was grown by MOCVD on $c$-plane sapphire. As shown in Figure 1, the $\mathrm{AlGaN} / \mathrm{AlN} / \mathrm{GaN}$ heterostructure consists of a $120 \mathrm{~nm}$ AlN nucleation layer, a $1.5 \mu \mathrm{m} \mathrm{GaN}$ semi-insulating layer, a $100 \mathrm{~nm}$ GaN channel layer, a $1 \mathrm{~nm}$ AlN interfacial layer, and a $20 \mathrm{~nm}$ $\mathrm{Al}_{0.2} \mathrm{Ga}_{0.8} \mathrm{~N}$ barrier layer.

The cross-sectional and plan-view TEM measurements are performed on the heterostructure sample. In Figure 2(a) the dark-field cross-sectional TEM image shows the dislocation distribution. The high-density dislocations near the substrate appear to be arranged irregularly. These dislocations are generated at the early stage of the growth when the neighboring three-dimensional (3D) islands coalesce due to the Volmer-Weber growth mode. After the islands coalesce and dislocations form, the growth mode switches from a 3D growth to a $2 \mathrm{D}$ growth. The threading dislocations vertically traverse from the AlN nucleation layer to the AlGaN barrier layer, which is accompanied by a decrease in the dislocation density. The areal density of dislocations in the AlGaN layer is estimated to be $1 \times 10^{9} \mathrm{~cm}^{-2}$ by counting dislocations from plan-view TEM images (see, e.g., Figure 2(b)). The highresolution cross-sectional TEM image (Figure 2(c)) shows abrupt $\mathrm{AlGaN} / \mathrm{AlN} / \mathrm{GaN}$ interfaces and a smooth $\mathrm{AlGaN}$ surface, further indicating a 2D growth mode of the AlGaN layer.

Dislocations can affect the 2DEG mobility in two ways: first, they induce local deformation of the crystal structure; secondly, the accumulated charges along the dislocation line can scatter electrons via Coulomb interactions. The effect of the local strain field on electron mobility is negligibly small compared with that of the Coulomb interactions [5]; therefore, only the Coulomb interactions are taken into account in the dislocation scattering in this study. Coulomb interactions are isotropic; thus, the calculations do not require determining the dislocation types.

Figure 3 shows a $3 \mu \mathrm{m} \times 3 \mu \mathrm{m}$ AFM image. The rootmean-square (RMS) surface roughness is $0.13 \mathrm{~nm}$, indicating a smooth surface. The statistical properties of surface roughness can be described by a correlation function, which is defined as [8]

$$
C(r)=\frac{1}{S} \int_{S} H\left(r^{\prime}\right) H\left(r^{\prime}+r\right) d r^{\prime},
$$

where $S$ is the sample area, $r^{\prime}$ is the position vector on the surface, $r$ is the relative distance, and $H\left(r^{\prime}\right)$ is the surface height at position $r^{\prime}$. The correlation function can be numerically calculated from the AFM measurement. But more often, two approximate analytical expressions, that is, for the Gaussian, $C(r)=\Delta^{2} \exp \left(-r^{2} / \Lambda^{2}\right)$, and exponential, $C(r)=$ $\Delta^{2} \exp (-r / \Lambda)$, are employed in theoretical calculations for mathematical convenience. Once the analytical form of the correlation function has been fixed, the surface roughness can be described by two parameters, that is, the RMS roughness $\Delta$ and the horizontal correlation length $\Lambda$ of surface heights. The $\Lambda$ is usually defined as a distance at which the value of correlation function drops to $e^{-1}=36.8 \%$ of the value at $r=0$ (here $e$ is the Neper number).

The normalized correlation function calculated from the AFM data is shown in Figure 4. The correlation length $\Lambda$ is determined to be $4.5 \mathrm{~nm}$ according to the definition above. In addition, the analytical Gaussian and exponential correlation functions with $\Lambda=4.5 \mathrm{~nm}$ are present in Figure 4 for comparison. It can be seen from Figure 4 that the exponential form more closely resemble the measured results than the Gaussian form. Therefore, the exponential correlation function is substituted for the measured one in the following scattering calculations.

Figure 5 shows the temperature-dependent Hall-effect measurements. The electron mobility monotonically increases with the decrease of the temperature and gradually saturates below $100 \mathrm{~K}$. The sheet carrier density, $N_{s}=1.05 \times 10^{13} \mathrm{~cm}^{-2}$, is almost temperature independent, indicating that the contribution of bulk carriers is quite small. The Hall-effect measurements exhibit obvious characteristics of the 2DEG formation in the AlGaN/AlN/GaN heterostructure. 


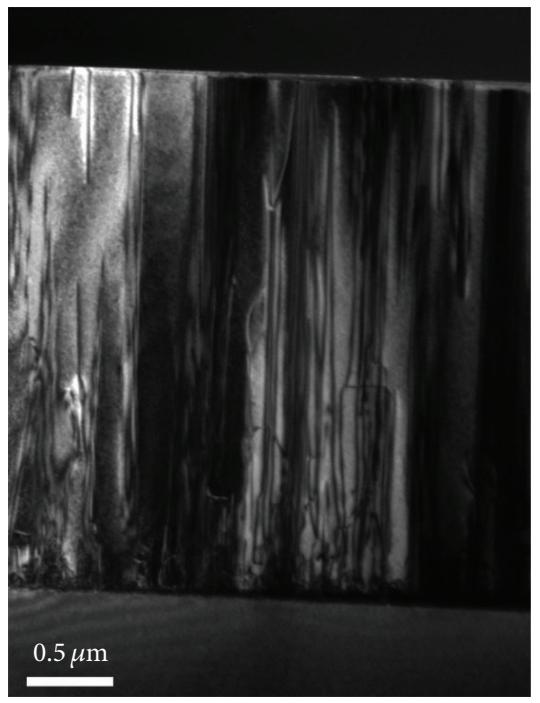

(a)

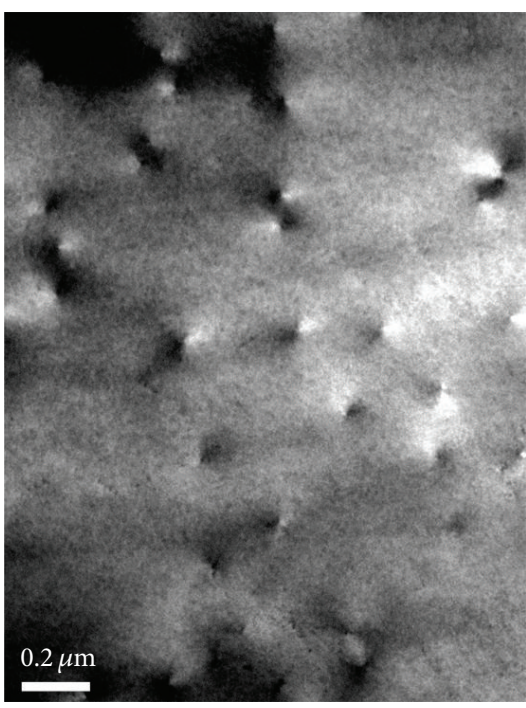

(b)

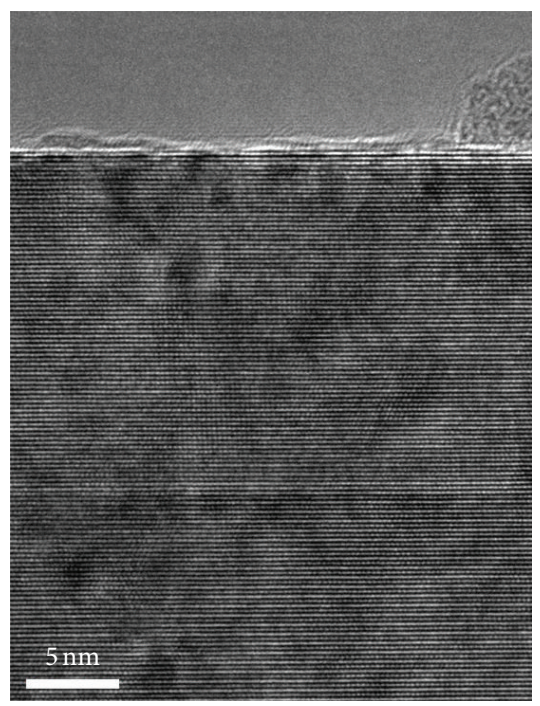

(c)

Figure 2: (a) Dark-field cross-sectional TEM image, (b) bright-field plan-view TEM image, and (c) high-resolution cross-sectional TEM image of the AlGaN/AlN/GaN heterostructure.

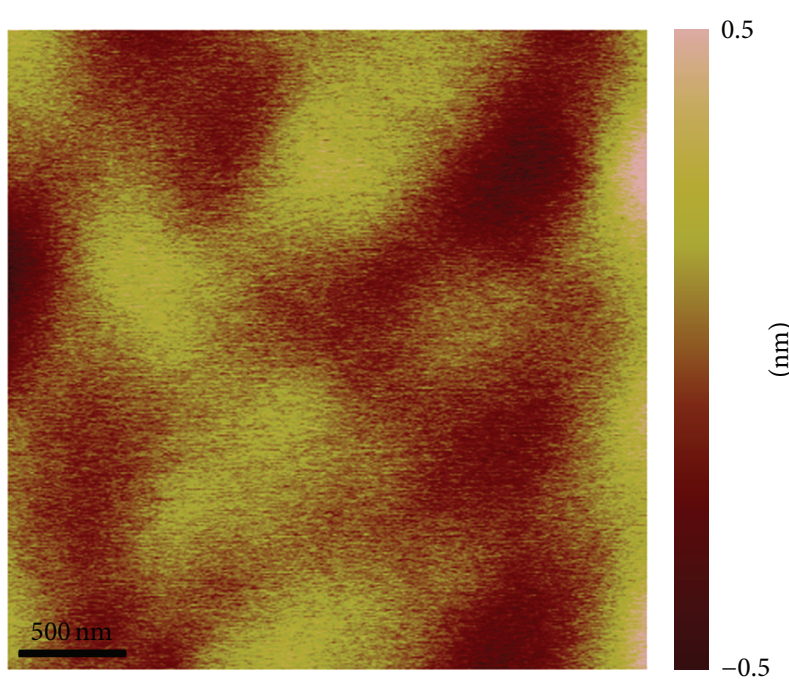

FIGURE 3: AFM image of the AlGaN/AlN/GaN heterostructure with an RMS roughness of $0.13 \mathrm{~nm}$.

\section{Theoretical Descriptions}

The scattering theories of the 2DEG system have been well developed by several authors [5, 13-19]. We give below a brief description of the analytical expressions for the momentum relaxation time $\tau_{i}(E)$ due to the important scattering mechanisms. The corresponding mobility component is calculated via $\mu_{i}=e \tau_{i} / m^{*}$; here $e$ is the electronic charge, and $m^{*}$ is the electron effective mass. The total mobility is calculated using Matthiessen's rule:

$$
\frac{1}{\mu_{\mathrm{TOT}}}=\sum_{i} \frac{1}{\mu_{i}}
$$

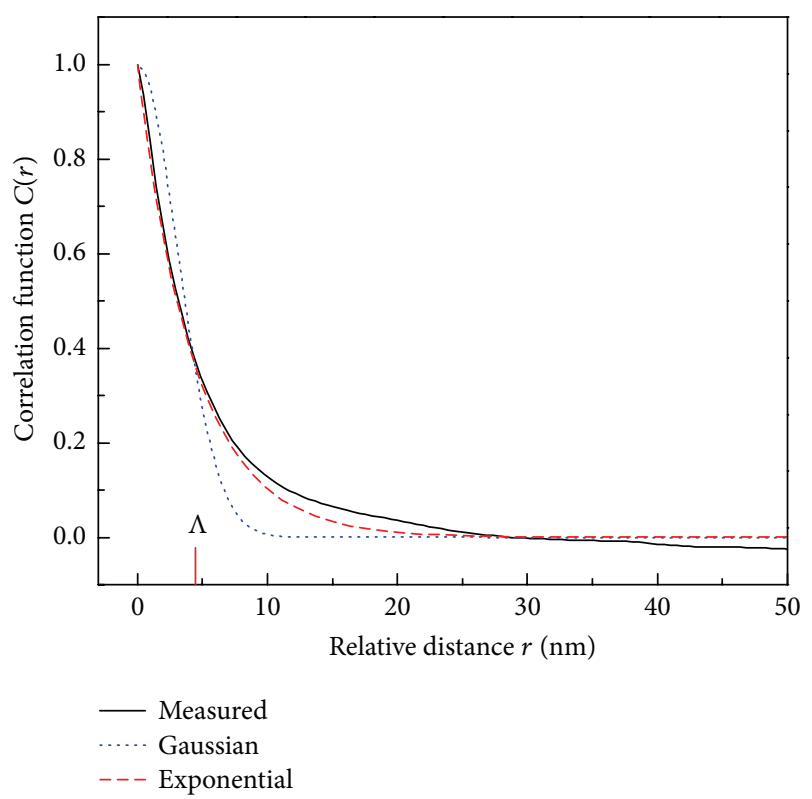

FIGURE 4: Normalized correlation function of surface roughness as a function of relative distance. The solid line is calculated from the AFM data, and the dashed and dotted lines represent the exponential and Gaussian correlation functions, respectively.

3.1. Dislocation Scattering (DIS). The matrix element for the Coulomb interactions of a single dislocation line is expressed as $[20]$

$$
\begin{aligned}
& \left|M_{\mathrm{DIS}}\right|^{2}=\frac{e^{2} \rho_{l}^{2}}{4 S \varepsilon_{0}^{2} \varepsilon_{s}^{2} q^{2}}\left\{\int\left[\int \exp \left(-q\left|z^{\prime}-z\right|\right) d z^{\prime}\right]\right. \\
& \left.\cdot|\xi(z)|^{2} d z\right\}^{2},
\end{aligned}
$$




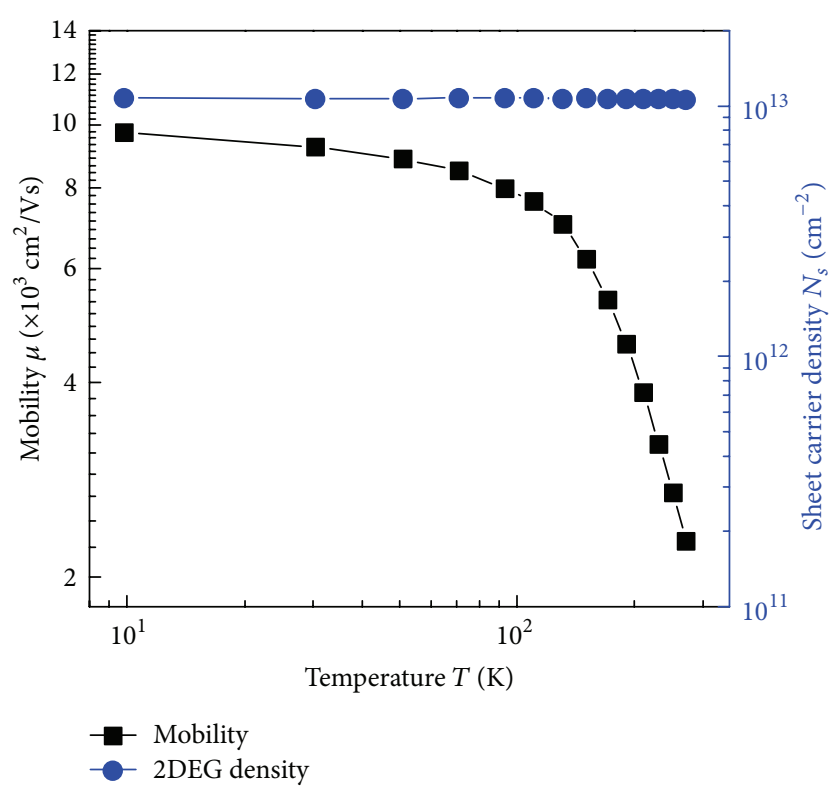

FIGURE 5: Mobility and sheet carrier density as a function of temperature for the $\mathrm{AlGaN} / \mathrm{AlN} / \mathrm{GaN}$ heterostructure.

where $q$ is the scattered wave vector, $\varepsilon_{0}$ is the permittivity of free space, $\varepsilon_{s}$ is the static permittivity, $\rho_{l}=e f / c$ is the linear charge density along the dislocation line, $f$ is the fraction of filled states, $c$ is the lattice constant along the wurtzite GaN [0001] direction, and $\xi(z)$ is the electron wave function. Here we set $f=1$; that is, there is one charge in every interval length of $c$ along the dislocation line.

The reciprocal momentum relaxation time for the DIS is given by

$$
\begin{aligned}
& \frac{1}{\tau_{\mathrm{DIS}}(E)}=\frac{N_{\mathrm{DIS}} m^{*} e^{2} \rho_{l}^{2}}{4 \pi \hbar^{3} \varepsilon_{0}^{2} \varepsilon_{s}^{2}} \\
& \cdot \int_{0}^{\pi}\left\{\int\left[\int \exp \left(-q\left|z^{\prime}-z\right|\right) d z^{\prime}\right]|\xi(z)|^{2} d z\right\}^{2} \\
& \cdot \frac{(1-\cos \theta)}{q^{2} \epsilon^{2}(q)} d \theta
\end{aligned}
$$

where $N_{\text {DIS }}$ is the dislocation density, $\hbar$ is the reduced Planck's constant, and $\theta$ is the angle between electron wave vectors before and after scattering. The wave vector $q$ is related to $\theta$ via $q=2 k \sin (\theta / 2) ; k$ is the electron wave vector. $\epsilon(q)$ is the screening function within the zero-temperature randomphase approximation [16].

3.2. Interface Roughness Scattering (IRS). Interface roughness induces fluctuations in the position of the barrier layer, leading to perturbations of the electron energy and wave function in the well layer. The effect of interface roughness on electron mobility is more important in narrower wells [12].
For an exponentially correlated surface, the matrix element for the IRS can be expressed as

$$
\left|M_{\text {IRS }}\right|^{2}=\frac{e^{4} \Delta^{2} \Lambda^{2} N_{s}^{2}}{2 S \varepsilon_{0}^{2} \varepsilon_{s}^{2}}\left(1+q^{2} \Lambda^{2}\right)^{-3 / 2}
$$

The reciprocal momentum relaxation time is given by

$$
\begin{aligned}
& \frac{1}{\tau_{\text {IRS }}(E)} \\
& \quad=\frac{e^{4} m^{*} \Delta^{2} \Lambda^{2} N_{s}^{2}}{2 \pi \hbar^{3} \varepsilon_{0}^{2} \varepsilon_{s}^{2}} \int_{0}^{\pi}\left(1+q^{2} \Lambda^{2}\right)^{-3 / 2} \frac{(1-\cos \theta)}{\epsilon^{2}(q)} d \theta .
\end{aligned}
$$

3.3. Charged Impurity Scattering (CIS). Most AlGaN/GaN heterostructures have been prepared without doping, but residual impurities, such as carbon, hydrogen, interstitial atoms, and vacancies, cannot be completely eliminated [21]. The neutral impurity scattering is quite small and consequently is neglected in this work. However, the CIS may dominate the electron mobility at low temperatures. The matrix element for the CIS is expressed as [12]

$$
\left|M_{\mathrm{CIS}}\right|^{2}=\frac{N(z)}{S}\left(\frac{e^{2} F_{c}(q, z)}{2 \varepsilon_{0} \varepsilon_{s} q}\right)^{2}
$$

where $N(z)$ is the distribution function of charged impurities. $F_{c}(q, z)$ is given by [12]

$$
F_{c}(q, z)=\int\left|\xi\left(z^{\prime}\right)\right|^{2} \exp \left(-q\left|z-z^{\prime}\right|\right) d z^{\prime} .
$$

Assuming that charged impurities are evenly distributed throughout the heterostructure at a density of $N_{\text {CIS }}$, the reciprocal momentum relaxation time is given by

$$
\begin{aligned}
& \frac{1}{\tau_{\mathrm{CIS}}(E)} \\
& \quad=\frac{e^{4} m^{*} N_{\mathrm{CIS}}}{4 \pi \hbar^{3} \varepsilon_{0}^{2} \varepsilon_{s}^{2}} \int_{0}^{\pi} \frac{\int\left|F_{c}(q, z)\right|^{2} d z}{q^{2} \epsilon^{2}(q)}(1-\cos \theta) d \theta .
\end{aligned}
$$

3.4. Phonon Scattering. Acoustic phonon scattering may dominate the electron mobility in the temperature range from several tens to two hundred of degrees Kelvin. Because wurtzite $\mathrm{GaN}$ is noncentrosymmetric, acoustic phonons can affect the electron mobility via deformation-potential (DP) and piezoelectric (PE) scatterings. In this study both DP and PE scatterings are taken into account. A detailed description of the acoustic phonon scattering can be found in $[13,15]$.

Polar optical (PO) phonon scattering dominates the electron mobility at high temperatures. The momentum relaxation time for $\mathrm{PO}$ phonon scattering is hardly strictly defined because the PO phonon scattering is inelastic and anisotropic. An accurate approach for studying PO phononlimited mobility $\left(\mu_{\mathrm{PO}}\right)$ is to directly solve the Boltzmann equation, but in that way one must carry out some really elaborate mathematical calculations. Here we use an approximate analytical expression to calculate $\mu_{\mathrm{PO}}$ [16].

The physical parameters used in the calculations are listed in Table 1. 
TABle 1: Parameters of $\mathrm{ZnO}$ employed in calculations. Here $m_{0}$ is the free electron mass.

\begin{tabular}{lcc}
\hline Parameter & Symbol (units) & Value \\
\hline Mass density & $\rho\left(\mathrm{kg} / \mathrm{m}^{-3}\right)$ & $6.15 \times 10^{3^{\mathrm{a}}}$ \\
Electron effective mass & $\mathrm{m}^{*}$ & $0.22 \mathrm{~m}_{0}^{\mathrm{b}}$ \\
Dielectric constant (high frequency) & $\varepsilon_{\infty}$ & $5.47^{\mathrm{b}}$ \\
Dielectric constant (low frequency) & $\varepsilon_{s}$ & $10.4^{\mathrm{b}}$ \\
LA-phonon velocity & $u_{l}(\mathrm{~m} / \mathrm{s})$ & $6.56 \times 10^{3^{\mathrm{a}}}$ \\
LO-phonon energy & $\hbar \omega_{\mathrm{PO}}(\mathrm{meV})$ & $91.2^{\mathrm{b}}$ \\
& $e_{15}\left(\mathrm{C} / \mathrm{m}^{2}\right)$ & $-0.3^{\mathrm{c}}$ \\
Piezoelectric tensor & $e_{31}\left(\mathrm{C} / \mathrm{m}^{2}\right)$ & $-0.33^{\mathrm{c}}$ \\
& $e_{33}\left(\mathrm{C} / \mathrm{m}^{2}\right)$ & $0.65^{\mathrm{c}}$ \\
Deformation constant & $D(\mathrm{eV})$ & $8.5^{\mathrm{b}}$ \\
\hline
\end{tabular}

${ }^{\mathrm{a}}[16],{ }^{\mathrm{b}}[20],{ }^{\mathrm{c}}[22]$.

\section{Fittings}

For AlGaN/AlN/GaN heterostructures, the AlN interfacial layer produces a potential barrier of height $2.1 \mathrm{eV}$ at the AlN/GaN interface [23], effectively preventing electron penetration into the AlGaN layer. Therefore, the wave function, $\xi(z)=\left(b^{3} / 2\right)^{1 / 2} z \exp (-b z / 2)$, proposed by Fang and Howard [24], is still a good approximation to the electron distribution along the growth direction for AlGaN/AlN/GaN heterostructures and consequently is employed in the scattering calculations. For the same reason alloy scattering is neglected in this study.

For the 2DEG in our heterostructure sample, the Fermi energy $E_{F}=\hbar k_{F}^{2} / 2 m^{*} \gg 2 k_{B} T$ over the entire temperature range up to $300 \mathrm{~K}$, where $k_{F}=\sqrt{2 \pi N_{s}}$ is the Fermi wave vector; thus, the $2 \mathrm{DEG}$ is highly degenerate even at room temperature [25]. In the fitting to the experimental data, therefore, it is justified to perform scattering calculations only at the Fermi energy.

Figure 6 shows a comparison between the theoretical and experimental results. The theoretical calculations are in good agreement with the experimental data. The measured values of the dislocation density and interface roughness, that is, $N_{\text {DIS }}=1 \times 10^{9} \mathrm{~cm}^{-2}, \Delta=0.13 \mathrm{~nm}$, and $\Lambda=4.5 \mathrm{~nm}$, are used in the fittings. Charged impurities are assumed to be evenly distributed throughout the heterostructure at a density of $N_{\text {CIS }}$. In the fittings only the $N_{\text {CIS }}$ is treated as an adjustable parameter and is determined to be $N_{\mathrm{CIS}}=6 \times 10^{17} \mathrm{~cm}^{-3}$. A comparable unintentional doping level is frequently found in the literatures $[16,26]$; thus, the fitting value of the charged impurity density is easy to reach experimentally and is acceptable.

The direct measurements of the defect parameters allow the evaluation of the relative importance of the extrinsic scattering mechanisms. It can be seen from Figure 6 that the CIS imposes the most severe limitation on low- and moderatetemperature mobilities, followed by the DIS and IRS. The mobilities limited by acoustic DP and PE scatterings are inversely proportional to the temperature. Acoustic scattering is relatively less important for the $\mathrm{AlGaN} / \mathrm{AlN} / \mathrm{GaN}$ sample, but this scattering mechanism will play a more important

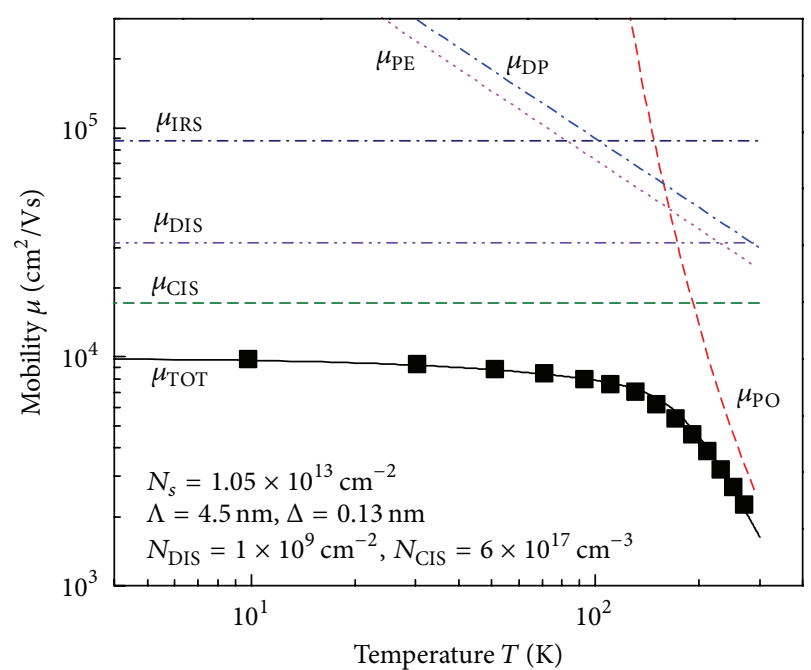

FIgURE 6: Temperature dependence of the mobility limited by individual scattering mechanisms including dislocation scattering $\left(\mu_{\mathrm{DIS}}\right)$, interface roughness scattering $\left(\mu_{\mathrm{IRS}}\right)$, charged impurity scattering $\left(\mu_{\mathrm{CIS}}\right)$, acoustic DP scattering $\left(\mu_{\mathrm{DP}}\right)$, acoustic PE scattering $\left(\mu_{\mathrm{PE}}\right)$, PO phonon scattering $\left(\mu_{\mathrm{PO}}\right)$, and all mentioned scattering mechanisms $\left(\mu_{\mathrm{TOT}}\right)$. The scattered symbols are the experimental data.

role in the heterostructures with perfect crystal quality. The mobility limited by $\mathrm{PO}$ phonons decreases exponentially with increasing temperature, and this scattering mechanism is dominant at temperatures above $200 \mathrm{~K}$.

Because the charged impurities dominate the electron transport at low and moderate temperatures, the calculation accuracies of DIS and IRS are more readily affected by measurement and model inaccuracies. But because of the larger distinction between the extrinsic scattering mechanisms, their relative importance would not be influenced by the inaccuracies.

\section{Conclusions}

In this study, we analyzed the dislocations and interface roughness in an $\mathrm{AlGaN} / \mathrm{AlN} / \mathrm{GaN}$ heterostructure via direct measurements and explained the temperature dependence of the Hall mobility using a theoretical model. The dislocation density is estimated to be $1 \times 10^{9} \mathrm{~cm}^{-2}$ by TEM. The correlation length $\Lambda$ and roughness height $\Delta$ are, respectively, determined to be 4.5 and $0.13 \mathrm{~nm}$ by AFM. The exponential correlation function, $C(r)=\Delta^{2} \exp (-r / \Lambda)$, gives a better description of the statistical properties of surface roughness than the Gaussian form and thus is employed in the theoretical model. The theoretical calculations are in good agreement with the experimental data. The quantitative measurements of the dislocation density and interface roughness allow the evaluation of the relative importance of each extrinsic scattering mechanism. It is found that the CIS is the most important scattering mechanism at low and moderate temperatures, followed by the DIS and IRS, whereas the PO phonon scattering is dominant above $200 \mathrm{~K}$. 


\section{Conflict of Interests}

The authors declare that there is no conflict of interests regarding the publication of this paper.

\section{Acknowledgments}

The authors would like to thank Chuansheng Ma of International Center for Dielectric Research for TEM measurements. This work was supported by the National Natural Science Foundation of China under Grant nos. 61176018 and 60876042 and the National High Technology Research and Development Program of China (863 Program) under Grant no. 2013AA03A101.

\section{References}

[1] M. Higashiwaki, S. Chowdhury, M.-S. Miao, B. L. Swenson, C. G. Van de Walle, and U. K. Mishra, "Distribution of donor states on etched surface of AlGaN/GaN heterostructures," Journal of Applied Physics, vol. 108, no. 6, Article ID 063719, 2010.

[2] E. Frayssinet, W. Knap, P. Lorenzini et al., "High electron mobility in AlGaN/GaN heterostructures grown on bulk GaN substrates," Applied Physics Letters, vol. 77, no. 16, pp. 2551-2553, 2000.

[3] V. P. Kladko, A. F. Kolomys, M. V. Slobodian et al., "Internal strains and crystal structure of the layers in $\mathrm{AlGaN} / \mathrm{GaN}$ heterostructures grown on a sapphire substrate," Journal of Applied Physics, vol. 105, no. 6, Article ID 063515, 2009.

[4] D. C. Look and J. R. Sizelove, "Dislocation scattering in GaN," Physical Review Letters, vol. 82, no. 6, pp. 1237-1240, 1999.

[5] F. Carosella and J.-L. Farvacque, "Effect of threading dislocations on carrier mobility in AlGaN/GaN quantum wells," Journal of Physics Condensed Matter, vol. 20, no. 32, Article ID 325210, 2008.

[6] X. Xu, X. Liu, X. Han et al., "Dislocation scattering in $\mathrm{Al}_{x} \mathrm{Ga}_{1-x} \mathrm{~N} / \mathrm{GaN}$ heterostructures," Applied Physics Letters, vol. 93, no. 18, Article ID 182111, 2008.

[7] S. M. Goodnick, D. K. Ferry, C. W. Wilmsen, Z. Liliental, D. Fathy, and O. L. Krivanek, "Surface roughness at the $\mathrm{Si}(100)$ $\mathrm{SiO}_{2}$ interface," Physical Review B, vol. 32, no. 12, pp. 8171-8189, 1985.

[8] G. Fishman and D. Calecki, "Influence of surface roughness on the conductivity of metallic and semiconducting quasi-twodimensional structures," Physical Review B, vol. 43, no. 14, pp. 11581-11585, 1991.

[9] L. Hsu and W. Walukiewicz, "Electron mobility in $\mathrm{Al}_{x} \mathrm{Ga}_{1-x} \mathrm{~N} /$ GaN heterostructures," Physical Review B, vol. 56, no. 3, pp. 1520-1528, 1997.

[10] A. Gold, "Theory of transport properties of the sixfolddegenerate two-dimensional electron gas at the $\mathrm{H}-\mathrm{Si}(111)$ surface," Physical Review B, vol. 82, no. 19, Article ID 195329, 2010.

[11] D. Zanato, S. Gokden, N. Balkan, B. K. Ridley, and W. J. Schaff, "The effect of interface-roughness and dislocation scattering on low temperature mobility of 2D electron gas in GaN/AlGaN," Semiconductor Science and Technology, vol. 19, no. 3, pp. 427432, 2004.

[12] J. M. Li, J. J. Wu, X. X. Han et al., "A model for scattering due to interface roughness in finite quantum wells," Semiconductor Science and Technology, vol. 20, no. 12, pp. 1207-1213, 2005.
[13] T. Kawamura and S. Das Sarma, "Phonon-scattering-limited electron mobilities in $\mathrm{Al}_{x} \mathrm{Ga}_{1-x}$ As/GaAs heterojunctions," Physical Review B, vol. 45, no. 7, pp. 3612-3627, 1992.

[14] T. Ando, A. B. Fowler, and F. Stern, "Electronic properties of two-dimensional systems," Reviews of Modern Physics, vol. 54, no. 2, pp. 437-672, 1982.

[15] P. J. Price, "Two-dimensional electron transport in semiconductor layers. I. Phonon scattering," Annals of Physics, vol. 133, no. 2, pp. 217-239, 1981.

[16] S. B. Lisesivdin, A. Yildiz, N. Balkan, M. Kasap, S. Ozcelik, and E. Ozbay, "Scattering analysis of two-dimensional electrons in $\mathrm{AlGaN} / \mathrm{GaN}$ with bulk related parameters extracted by simple parallel conduction extraction method," Journal of Applied Physics, vol. 108, no. 1, Article ID 013712, 2010.

[17] D. N. Quang, N. H. Tung, V. N. Tuoc, N. V. Minh, H. A. Huy, and D. T. Hien, "Quantum and transport lifetimes due to roughness-induced scattering of a two-dimensional electron gas in wurtzite group-III-nitride heterostructures," Physical Review B, vol. 74, no. 20, Article ID 205312, 2006.

[18] A. Asgari, S. Babanejad, and L. Faraone, "Electron mobility, Hall scattering factor, and sheet conductivity in AlGaN/AlN/GaN heterostructures," Journal of Applied Physics, vol. 110, no. 11, Article ID 113713, 2011.

[19] H. Tokuda, J. Yamazaki, and M. Kuzuhara, "High temperature electron transport properties in $\mathrm{AlGaN} / \mathrm{GaN}$ heterostructures," Journal of Applied Physics, vol. 108, no. 10, Article ID 104509, 2010.

[20] M. N. Gurusinghe, S. K. Davidsson, and T. G. Andersson, "Two-dimensional electron mobility limitation mechanisms in $\mathrm{Al}_{x} \mathrm{Ga}_{1-x} \mathrm{~N} / \mathrm{GaN}$ heterostructures," Physical Review B, vol. 72, no. 4, Article ID 045316, 2005.

[21] A. Ishibashi, H. Takeishi, M. Mannoh, Y. Yabuuchi, and Y. Ban, "Residual impurities in $\mathrm{GaN} / \mathrm{Al}_{2} \mathrm{O}_{3}$ grown by metalorganic vapor phase epitaxy," Journal of Electronic Materials, vol. 25, no. 5, pp. 799-803, 1996.

[22] A. D. Bykhovski, B. L. Gelmont, and M. S. Shur, "Elastic strain relaxation and piezoeffect in GaN-AlN, GaN-AlGaN and GaNInGaN superlattices," Journal of Applied Physics, vol. 81, no. 9, pp. 6332-6338, 1997.

[23] A. Janotti and C. G. van de Walle, "Absolute deformation potentials and band alignment of wurtzite $\mathrm{ZnO}, \mathrm{MgO}$, and CdO," Physical Review B, vol. 75, no. 12, Article ID 121201, 2007.

[24] F. F. Fang and W. E. Howard, "Negative field-effect mobility on (100) Si surfaces," Physical Review Letters, vol. 16, no. 18, pp. 797799, 1966.

[25] R. Grill and G. H. Döhler, "Effect of charged donor correlation and Wigner liquid formation on the transport properties of a two-dimensional electron gas in modulation $\delta$-doped heterojunctions," Physical Review B, vol. 59, no. 16, pp. 10769-10777, 1999.

[26] T. Zhu and R. A. Oliver, "Unintentional doping in GaN," Physical Chemistry Chemical Physics, vol. 14, no. 27, pp. 9558-9573, 2012. 

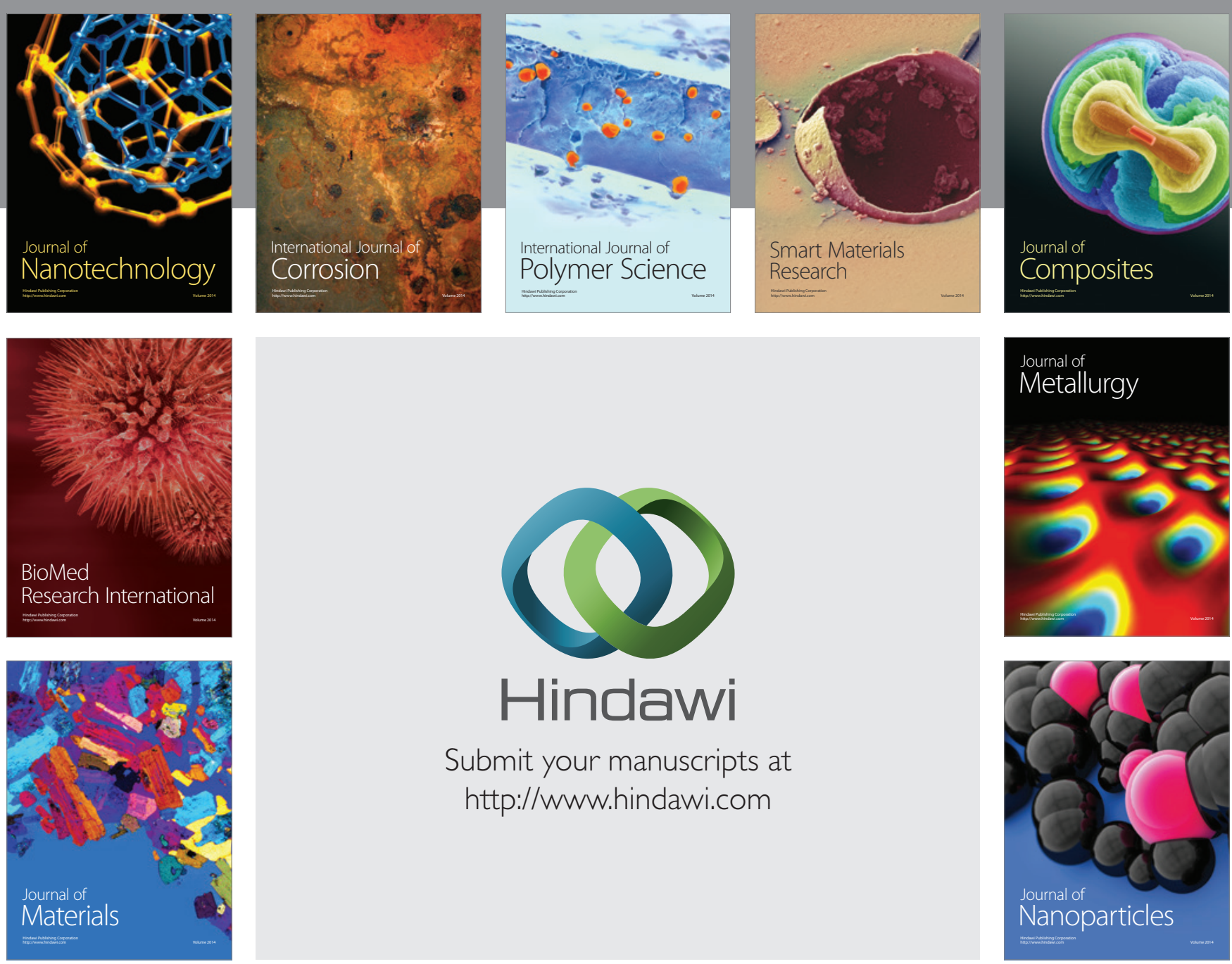

Submit your manuscripts at http://www.hindawi.com
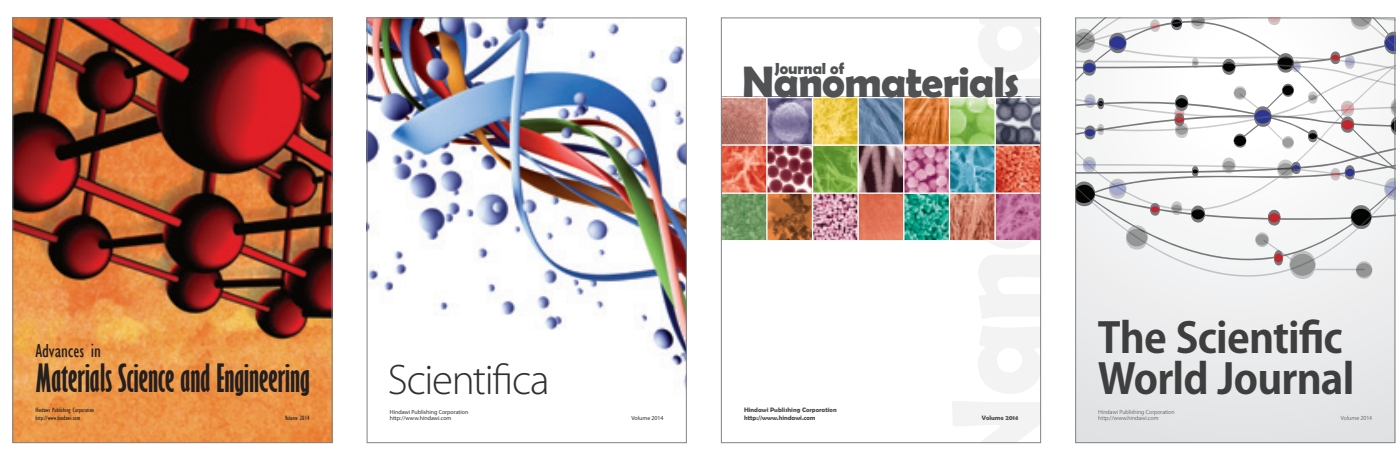

\section{The Scientific World Journal}
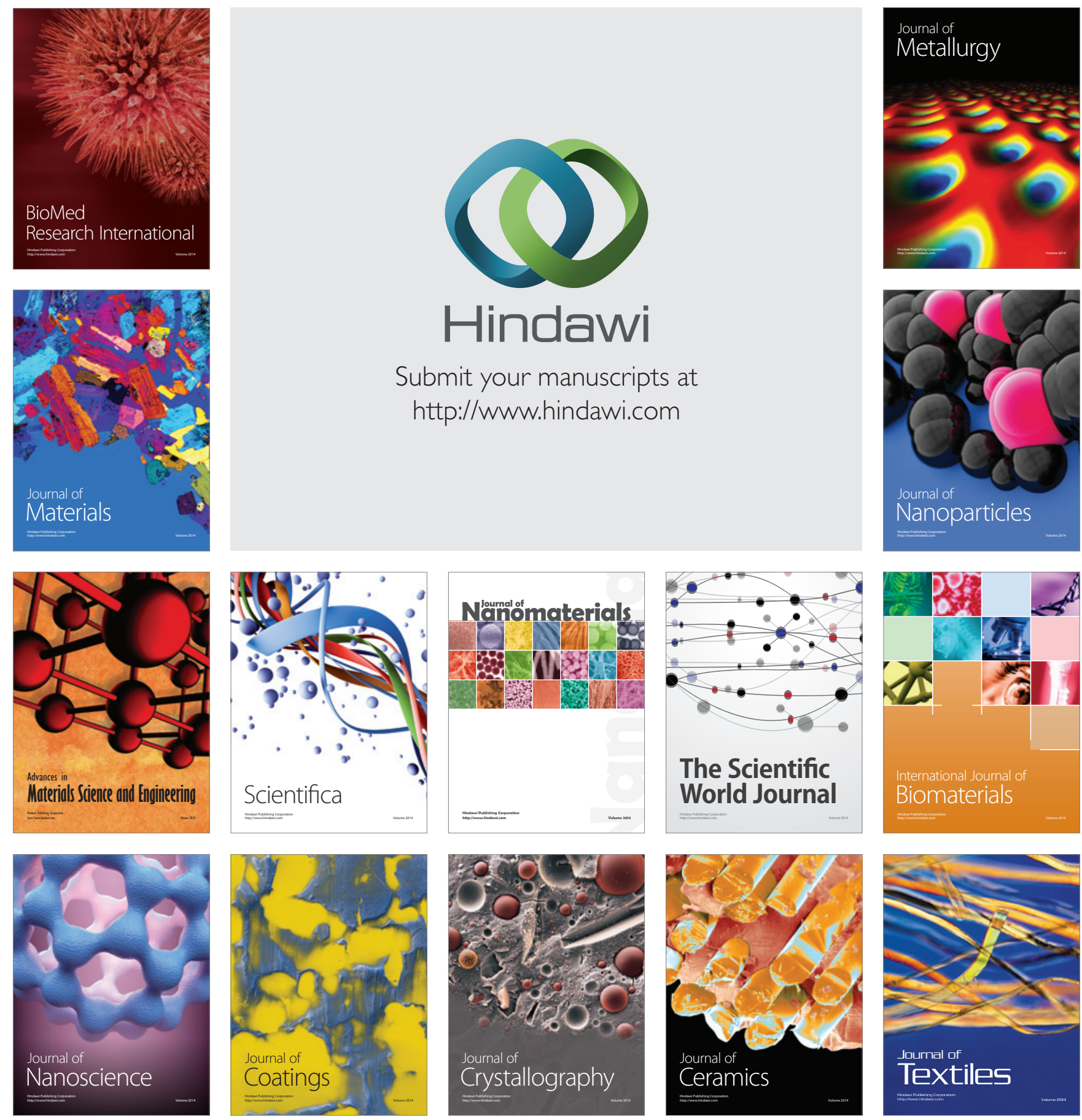